Sādhanā, Vol. 9, Part 4, December 1986, pp. 371-383. (C) Printed in India.

\title{
A half-plane model for analysis of stress in the blade of a spade
}

\author{
P K SHARMA, G S SEKHON anḍ S V PATWARDHAN* \\ Applied Mechanics Department, and \\ *Centre for Rural Development and Appropriate Technology, Indian \\ Institute of Technology, New Delhi 110 029, India \\ MS received 16 April 1984; revised 28 July 1985
}

\begin{abstract}
The present work deals with the state of stress produced in the blade of a spade as a result of an external load acting around its middle. Such loadings can occur when the cutting edge of a spade encounters a hard obstacle such as a pebble or a boulder. It is shown that a half-plane model can simulate the situation. Stress distributions have been obtained corresponding to five types of external load, namely, concentrated normal, distributed normal, concentrated tangential, distributed tangential and combined normal and tangential. A discussion on the significance of the results is also included.
\end{abstract}

Keywords. Spade; stress analysis; half-plane model.

\section{Introduction}

The blade of a spade should neither fracture, nor deform plastically during its intended life. When a 'spade' is used on soil free from boulders, the loads on the blade are usually not capable of causing plastic deformation in the blade. However, in case the soil does contain boulders and the blade happens to strike one or several of them, large stresses could develop in the blade which might cause either plastic deformation or progressive fatigue failure. That is why an analysis of the distribution of stress due to loading, arising from the impact of a blade against a boulder, is of considerable practical importance in understanding the causes of failure of the blade. In this paper, we present a model for the analysis of stress in the blade of a 'spade'. The model deals with the situation when the loading is at or near the middle portion of the leading edge of the blade, that is, well away from the ends.

\section{The proposed model}

When the leading edge of a blade of a 'spade' strikes against a rigid or stiff obstacle whose width $C$ is small as compared to width $b$ of the blade, the loading on the 
blade is confined to a portion of the edge whose length is much smaller than that of the edge itself. Also, since the thickness of the edge is small as compared to either $b$ or $C$, it is safe to assume the loading to be uniform across the thickness of the edge. Further, let the portion of the edge that strikes against the obstacle be quite removed from either corner. This situation corresponds to a typical half-plane problem of the theory of elasticity with the blade of the 'spade' constituting the halfplane.

The blade is idealized as a plate made of a homogeneous, isotropic, perfectly elastic material (figure 1 ). The thickness of the plate, $2 h$, is considered to be small as compared to its other dimensions. A uniformly distributed load $Q$ acts at a uniform inclination angle $\phi$ on a portion of length $C$ of the edge. As a result, the normal and tangential stresses $p$ and $t$ are uniformi over the length $C$ and are given by

$$
\begin{aligned}
& p=Q \sin \phi / t C=q \cos \phi, \\
& t=Q \sin \theta / t C=q \sin \phi,
\end{aligned}
$$

where $q=Q /(t \times c)$ is the intensity of the external load. It is desired to obtain stress distribution in the plate on account of the given load. Inertia effects are neglected.

\section{Analysis}

The above plane-stress problem can be split up into two parts - one corresponding to the uniform normal and the other to the uniform tangential stress acting on a portion of the edge of the haif-plane. Also it is convenient to obtain the solution for a distributed external load from that of a concentrated line load by integration (Timoshenko \& Goodier 1951; Saada 1974). The procedure is briefly discussed below.

\subsection{Stress state due to a concentrated normal line-load}

Consider a normal line-load $P$ (force per unit width) acting on the edge of a plate constituting the half-plane. Let the origin $O$ of the $(x, y)$ and $(r, \theta)$ coordinates be placed at the point on the edge where the external load is acting (figure 2 ). Because

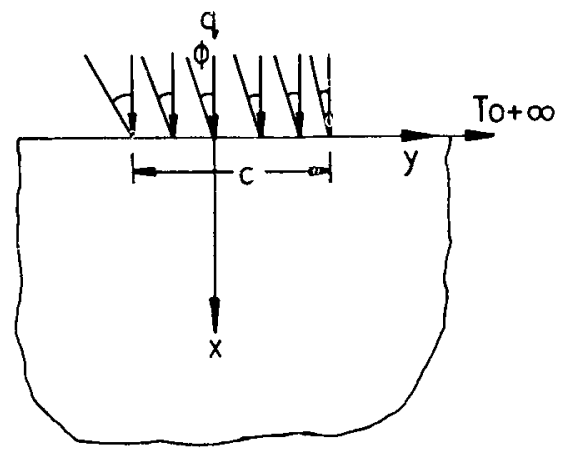

Figure 1. The proposed half-plane model. 


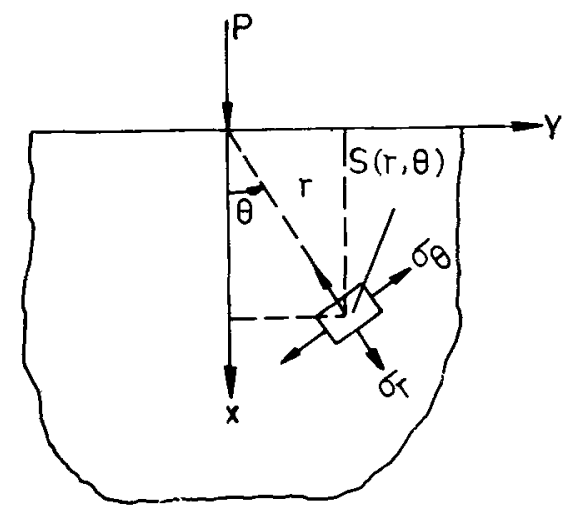

Figure 2. A concentrated normal line-load acting on the edge of the half-plane.

the thickness of the plate is small, $\sigma_{z}, \tau_{r z}$ and $\tau_{\theta z}$ vanish everywhere, leaving only three non-zero components of stress, namely, $\sigma_{r}, \sigma_{\theta}$ and $\tau_{r \theta}$.

The two non-trivial equations of equilibrium in this case are

$$
\begin{aligned}
& \frac{1}{r} \frac{\partial\left(r \sigma_{r}\right)}{\partial r}+\frac{1}{r} \frac{\partial \tau_{r \theta}}{\partial \theta}-\frac{\sigma_{\theta}}{r}=0, \\
& \frac{1}{r^{2}} \frac{\partial\left(r^{2} \tau_{r \theta}\right)}{\partial r}+\frac{1}{r} \frac{\partial \sigma_{\theta}}{\partial \theta}=0 .
\end{aligned}
$$

The above equations are satisfied identically if the stresses are derived from the so-called Airy stress function $\phi(r, \theta)$ such that

$$
\begin{aligned}
& \sigma_{r}=\frac{1}{r} \frac{\partial \phi}{\partial r}+\frac{1}{r^{2}} \frac{\partial^{2} \phi}{\partial \theta^{2}} \\
& \sigma_{\theta}=\frac{\partial^{2} \phi}{\partial r^{2}} \\
& \tau_{r \theta}=-\frac{\partial}{\partial r}\left(\frac{1}{r} \frac{\partial \phi}{\partial \theta}\right) .
\end{aligned}
$$

Also the compatibility condition, namely,

$$
\left(\frac{\partial^{2}}{\partial r^{2}}+\frac{1}{r} \frac{\partial}{\partial r}+\frac{1}{r^{2}} \frac{\partial^{2}}{\partial \theta^{2}}\right)\left(\sigma_{r}+\sigma_{\theta}\right)=0
$$

is satisfied if $\sigma_{r}$ and $\sigma_{\theta}$ are derived from the above function $\phi(r, \theta)$.

Using $\phi=-(P / \pi) r \theta \sin \theta$ and (4), the following expressions for stress distribution are obtained (Timoshenko \& Goodier 1951; Saada 1974):

$$
\begin{aligned}
& \sigma_{r}=-(2 P / \pi)(\cos \theta / r), \\
& \sigma_{\theta}=\tau_{r \theta}=0 .
\end{aligned}
$$

In other words, the stress state is one of uniaxial compression in the radial direction through the point of application of the load. It can be shown that the 
resultant load in the $x$-direction due to the radial stress $\sigma_{r}$ on any semi circular portion of radius $r$ and centre $O$ is equal to the external load $P$. Since $r$ can be made as small as desired, it follows that (6) constitutes the desired solution to the problem of a concentrated normal line load acting on the half-plane. Also it is found from (6) that the stress $\sigma_{r}$, at any point $S(r, \theta)$, is inversely proportional to the radial distance $r$, tending to a large value as $r$ tends to zero. Let $\sigma_{\theta}$ be the yield stress of the material of the blade in uniaxial compression. Applying (6) and neglecting any redistributive effects of plastic deformation, the radial distance $r_{p}$ of the elasto-plastic boundary is obtained as

$$
r_{p}=\left(2 P / \pi \sigma_{0}\right) \cos \theta=r_{p_{0}} \cos \theta .
$$

If at any point $S(r, \theta), r$ is less than $r_{p}$, plastic deformation would occur at that point. Figure 3 shows the typical circular plastic zone resulting from a concentrated normal load. It would be noted that the greater the magnitude of the external load or the smaller the yield stress of the material, the larger the extent of the plastic region.

The components of stress in the cylindrical coordinates $(r, \theta)$ can be transformed to cartesian coordinates by making use of the relations

$$
\begin{aligned}
& \sigma_{x}=\sigma_{r} \cos ^{2} \theta+\sigma_{\theta} \sin ^{2} \theta-\tau_{r \theta} \sin 2 \theta, \\
& \sigma_{y}=\sigma_{r} \sin ^{2} \theta+\sigma_{\theta} \cos ^{2} \theta+\tau_{r \theta} \sin 2 \theta, \\
& \tau_{x y}=\left(\sigma_{r}-\sigma_{\theta}\right) / 2 \sin 2 \theta+\tau_{r \theta} \cos 2 \theta
\end{aligned}
$$

where $\theta$ is measured in the anticlockwise direction from the $x$-axis. Since $\sigma_{\theta}$ and $\tau_{r \theta}$ are zero in the present case, the above relations simplify to

$$
\begin{aligned}
\sigma_{x} & =\sigma_{r} \cos ^{2} \theta=-(2 P / \pi r) \cos ^{3} \theta, \\
\sigma_{y} & =\sigma_{r} \sin ^{2} \theta=-(2 P / \pi r) \cos \theta \sin ^{2} \theta \\
\tau_{x y} & =\left(\sigma_{r} \sin 2 \theta\right) / 2=-(2 P / \pi r) \cos ^{2} \theta \cdot \sin \theta .
\end{aligned}
$$

Also from figure (2)

$$
\begin{aligned}
r & =\left(x^{2}+y^{2}\right)^{1 / 2}, \\
\cos \theta & =x /\left(x^{2}+y^{2}\right)^{1 / 2}, \\
\text { and } \quad \sin \theta & =y /\left(x^{2}+y^{2}\right)^{1 / 2} .
\end{aligned}
$$

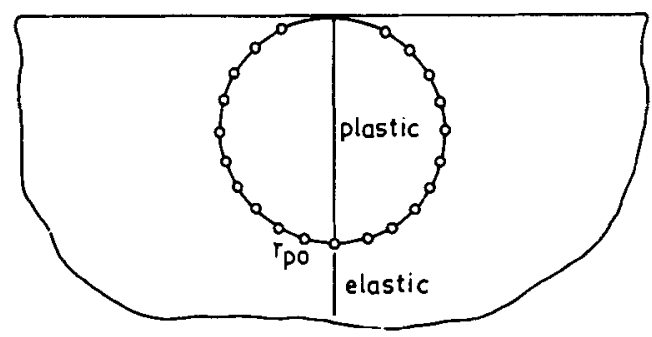

Figure 3. Extent of the plastic zone due to a concentrated normal line-load.

$$
\left(r_{p_{0}}=2 P / \pi \sigma_{0}\right)
$$


Theretore the components of plane stress expressed in cartesian coordinates at any point $S(x, y)$ are obtained as

$$
\begin{aligned}
\sigma_{x} & =-(2 P / \pi) \times\left[x^{3} /\left(x^{2}+y^{2}\right)^{2}\right], \\
\sigma_{y} & =-(2 P / \pi)\left[x y^{2} /\left(x^{2}+y^{2}\right)^{2}\right], \\
\tau_{x y} & =-(2 P / \pi)\left[x^{2} y /\left(x^{2}+y^{2}\right)^{2}\right] .
\end{aligned}
$$

\subsection{Stress state due to distributed normal pressure}

Figure 4 shows a uniform pressure $p$ acting over a length $C$ of the edge of the half-plane. Consider first the effect at point $S$ due to the normal load $\mathrm{d} P=p \mathrm{~d} \xi$ acting over an infinitesimal length $\mathrm{d} \xi$ centred around $O^{\prime}$ on the edge of the half-plane. Let the coordinates of the point $S$ be $(r, \theta)$ with respect to $O^{\prime}$. The component of stress at any point $S$ due to the normal load $\mathrm{d} P$ is then obtained with the help of (8) as

$$
\Delta \sigma_{x}=-(2 \mathrm{~d} P / \pi r) \cos ^{3} \theta=-2 p(\mathrm{~d} \xi / \pi r) \cos ^{3} \theta
$$

But

so

$$
\mathrm{d} \xi=r(\mathrm{~d} \theta / \cos \theta),
$$

$$
\Delta \sigma_{x}=-(2 p / \pi) \cos ^{2} \theta \mathrm{d} \theta .
$$

Hence the normal stress component $\sigma_{x}$ due to the normal pressure $p$ over the length $C$ is given by the integral

$$
\begin{aligned}
\sigma_{x} & =\int_{\theta_{1}}^{\theta_{2}} \Delta \sigma_{x}=-(2 p / \pi) \int_{\theta_{1}}^{\theta_{2}} \cos ^{2} \theta \mathrm{d} \theta \\
& =-(p / 2 \pi)\left[2\left(\theta_{2}-\theta_{1}\right)+\left(\sin 2 \theta_{2}-\sin 2 \theta_{1}\right)\right] .
\end{aligned}
$$

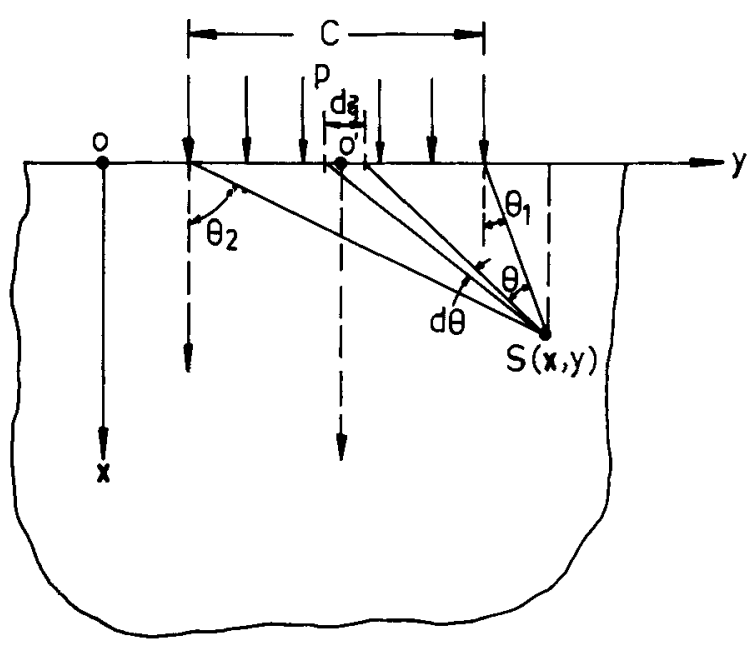

Figure 4. Normal pressure $p$ acting over a portion of the edge of a half-plane. 
Similarly, it can be shown (Saada 1974) that at any point $S(x, y)$

$$
\begin{aligned}
\sigma_{y} & =-(p / 2 \pi)\left[2\left(\theta_{2}-\theta_{1}\right)-\left(\sin 2 \theta_{2}-\sin 2 \theta_{1}\right)\right], \\
\tau_{x y} & =-(p / 2 \pi)\left(\cos 2 \theta_{1}-\cos 2 \theta_{2}\right) .
\end{aligned}
$$

The principal directions are given by the relation

$$
\tan 2 \beta=2 \tau_{x y} /\left(\sigma_{x}-\sigma_{y}\right)
$$

Substituting from (12) in (13), we find

$$
\tan 2 \beta=-\tan \left(\theta_{1}+\theta_{2}\right) \text {. }
$$

So $\beta$ has two roots $\beta_{1}$ and $\beta_{2}$ such that

$$
\beta_{1}=90^{\circ}-\left(\theta_{1}+\theta_{2}\right) / 2 \text {, }
$$

and

$$
\beta_{\mid \overrightarrow{\mid}}=180^{\circ}-\left(\theta_{1}+\theta_{2}\right) / 2
$$

Let $x^{\prime}, y^{\prime}$ be a set of orthogonal coordinates rotated by an angle $\beta$ anticlockwise with respect to the $x$ and $y$ axes. Applying the transformation rule

$$
\sigma_{x^{\prime} y^{\prime}}=\sigma_{x} \sin ^{2} \beta+\sigma_{y} \cos ^{2} \beta+\tau_{x y} \sin 2 \beta,
$$

and (15), the expressicns for principal stresses $\sigma_{1}$ and $\sigma_{2}$ are given by

$$
\sigma_{1}=-(p / \pi)(\alpha+\sin \alpha)
$$

and $\quad \sigma_{2}=-(p / \pi)(\alpha-\sin \alpha)$,

where $\alpha=\theta_{2}-\theta_{1}$.

For all points on the edge lying within the loaded portion, $\alpha=\pi, \beta_{1}=0^{\circ}$, $\beta_{2}=90^{\circ}$,

$$
\sigma_{1}=\sigma_{2}=-p
$$

The maximum shear stress in the $x-y$ plane, $\tau_{12}$, is given by

$$
\tau_{12}=\frac{\sigma_{1}-\sigma_{2}}{2}=-(p / \pi) \sin \alpha .
$$

This is maximum when $\alpha=90^{\circ}$, which is the case for all points lying on a semi-circle with radius $C / 2$ and the centre on the midpoint of the loaded portion of the edge. The maximum shear stresses in the $x-y$ and $y-z$ planes, $\tau_{13}$ and $\tau_{23}$ respectively, are however both equal to $-p / 2$.

Áccording to von Mises yield criterion (Caddle 1980),

$$
\begin{aligned}
\vec{\sigma} & =\left(\sigma_{1}^{2}+\sigma_{2}^{2}-\sigma_{1} \sigma_{2}\right)^{1 / 2} \\
& =\sigma_{0},
\end{aligned}
$$

where $\sigma_{0}$ is the yield stress of material under uniaxial tension. We may approximately obtain (by neglecting the redistributive effects on stress of plastic deformation) the extent of the plastic zone by applying the above criterion to (15)

$$
p / \sigma_{0}=\pi /\left[\alpha^{2}+3 \sin ^{2} \alpha\right]^{1 / 2} .
$$

Figure 5 shows the plastic region according to the above criterion for different values of $p / \sigma_{0}$. It should be noted that the greater the normal pressure $p$ or the smalier the yield stress $\sigma_{0}$, the larger he size of the plastic zone. Also the plastic 


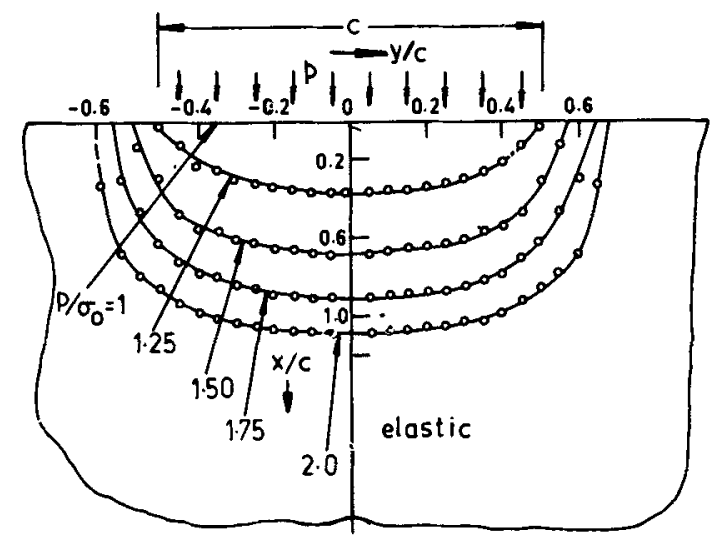

Figure 5. Variation of the plastic zone with normal pressure.

zone is symmetrical about the neutral axis passing through the centre of the loaded portion of the half-plane.

\subsection{Stress distribution due to a concentrated tangential line load}

Figure 6 shows a concentrated tangeritial line load acting on a half-plane. The Airy-stress function $\phi$ appropriate to this type of load on the half-plane is given by

$$
\phi=(T / \pi) r \theta \cos \theta
$$

Using (4), the stress components in cylincirical polar coordinates are obtained as

$$
\begin{aligned}
& \sigma_{r}=-(2 \pi / \pi r) \sin \theta, \\
& \sigma_{\theta}=0, \\
& \tau_{r \theta}=0 .
\end{aligned}
$$

The state of stress is uniaxial, being tensile for all points of the half-plane situated to the left of a vertical axis passing through the point of application of the load, and vice-versa. It can be shown that the resultant load in the $y$-direction due to the radial stress $\sigma_{r}$ on any semi-circular portion of radius $r$ and centre $O$ equals the

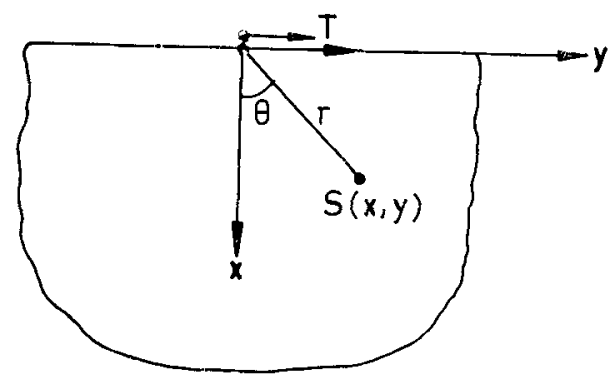

Figure 6. A concentrated tangential load $T$ acting on the half-plane. 
external load $T$ in that direction. Equation 18 shows that $\theta_{r}$ varies inversely with $r$, tending to a large value as $r$ tends to zero.

Applying (21), the radial distance $r p$ of the elasto-plastic boundary is approximately given by

$$
\begin{aligned}
r_{p} & =\left(2 T / \pi \sigma_{0}\right) \sin \theta \\
& =r_{p_{0}} \sin \theta .
\end{aligned}
$$

Figure 7 shows the typical shape of the plastic zone. In contrast to the case when a normal line-load acts on the half-plane, a concentrated tangential line-load produces two distinct semi-circular plastic zones. The one trailing the load is tensile in character and that in front of the applied load is compressive. The sizes of the two plastic zones increase with increase in load $T$ and decrease in the yield stress $\sigma_{0}$.

The components of stress in cartesian coordinates corresponding to the state of stress given by (21) are found to be

and

$$
\begin{aligned}
& \sigma_{x}=-(2 T / \pi r) \sin \theta \cos ^{2} \theta, \\
& \sigma_{y}=-(2 T / \pi r) \sin ^{3} \theta,
\end{aligned}
$$

$$
\tau_{x y}=-(2 T / \pi r) \sin ^{2} \theta \cos \theta
$$

Using (10), the above expressions can be rewritten as

$$
\begin{aligned}
\sigma_{x} & =-(2 T / \pi)\left[x^{2} y /\left(x^{2}+y^{2}\right)^{2}\right] \\
\sigma_{y} & =-(2 T / \pi)\left[y^{3} /\left(x^{2}+y^{2}\right)^{2}\right] \\
\tau_{x y} & =-(2 T / \pi)\left[x y^{2} /\left(x^{2}+y^{2}\right)^{2}\right]
\end{aligned}
$$

\subsection{Stress distribution due to a distributed tangential stress}

Following the procedure for the distributed normal stress case utilised in $\$ 3.2$, the stress distribution caused by a distributed tangential stress (figure 8 ) can also be obtained through integration. The normal stress $\Delta \sigma_{x}$ at a point $S$ caused by a tangential line-load $\mathrm{d} T=t \mathrm{~d} \xi$ acting over an infinitesimal length $\mathrm{d} \xi$ centred around $O^{\prime}$ on the edge of the half-plane is found with the help of (21) as

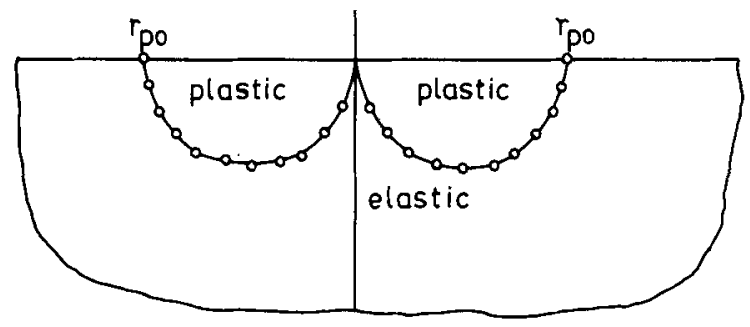

Figure 7. Extent of the plastic zones due to a tangential line-load.

$$
\left(r_{p_{0}}=2 T / \pi \sigma_{0}\right)
$$




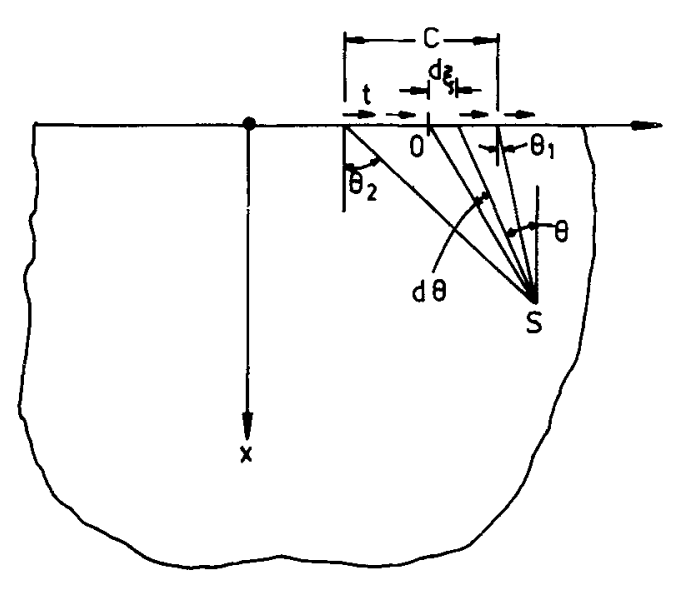

$$
\begin{aligned}
\Delta \sigma_{x} & =-(2 \mathrm{~d} T / \pi r) \sin \theta \cos ^{2} \theta \\
& =-2 t(\mathrm{~d} \xi / \pi r) \sin \theta \cos ^{2} \theta \\
& =-(2 t / \pi) \sin \theta \cos \theta \mathrm{d} \theta
\end{aligned}
$$

Therefore, the net stress $\sigma_{x}$ at point $S$ will be given by

$$
\begin{aligned}
& \sigma_{x}=\int_{\theta_{1}}^{\theta_{2}} \Delta \sigma_{x}=-\frac{2 t}{\pi} \int_{\theta_{1}}^{\theta_{2}} \sin \theta \cos \theta \mathrm{d} \theta, \\
& \sigma_{x}=-\frac{t}{2 \pi}\left(\cos 2 \theta_{1}-\cos 2 \theta_{2}\right) .
\end{aligned}
$$

Similarly it is found that

$$
\sigma_{y}=-\frac{2 t}{\pi}\left[2 \log _{e}\left(\frac{\sec \theta_{2}}{\sec \theta_{1}}\right)+\frac{1}{4}\left(\cos 2 \theta_{2}-\cos 2 \theta_{1}\right)\right],
$$

and

$$
\tau_{x y}=-\frac{t}{2 \pi}\left[2\left(\theta_{2}-\theta_{1}\right)-\left(\sin 2 \theta_{2}-\sin 2 \theta_{1}\right)\right] .
$$

For all points on the edge lying within the loaded portion

$$
\begin{aligned}
& \partial_{1}=-\pi / 2, \quad \theta_{2}=\pi / 2, \quad \tau_{x y}=-t, \\
& \sigma_{x}=0, \quad \text { and } \sigma_{y}=0 .
\end{aligned}
$$

According to von Mises yield criterion,

$$
\bar{\sigma}=(2)^{-1 / 2}\left[\left(\sigma_{x}-\sigma_{y}\right)^{2}+\sigma_{y}^{2}+\sigma_{x}^{2}+6 \tau_{x v}^{2}\right]^{1 / 2},
$$

where $\sigma_{0}$ is the yield stress of material under uniaxial tension. Substituting the values of $\sigma_{x} ; \sigma_{y}$ and $\tau_{x y}$ from (22)-(24) and neglecting any redistributive effects of plastic deformation, the extents of the plastic zone due to different values of dis- 
tributed tangential stress are as shown in figure 9. It is found that as the nondimensionalized stress $t / \sigma_{0}$ increases, the size of the plastic zone also increases. Also, the maximum penetration of the plastic zone occurs at a distance of about $0.2 C$ from the middle of the loaded portion of the edge.

\subsection{Combined normal and tangential loading}

When both a distributed normal stress $p$ and a distributed tangential stress $t$ are operative on a portion of the length $C$ of the edge of a half-plane, use may be made of the principle of superposition to obtain the components of stress at any point $S(x, y)$. Hence, we have from (12) and (23)

$$
\begin{aligned}
\sigma_{x}= & -(p / 2 \pi)\left[2\left(\theta_{2}-\theta_{1}\right)+\left(\sin 2 \theta_{2}-\sin 2 \theta_{1}\right)\right. \\
& \left.-(t / 2 \pi) \cos 2 \theta_{1}-\cos 2 \theta_{2}\right], \\
\sigma_{y}= & -(p / 2 \pi)\left[2\left(\theta_{2}-\theta_{1}\right)-\left(\sin 2 \theta_{2}-\sin 2 \theta_{1}\right)\right] \\
& -\underset{*}{(2 t / \pi)}\left[\log _{\mathrm{e}}\left(\cos \theta_{1} / \cos \theta_{2}\right) \frac{1}{4}\left(\cos 2 \theta_{2}-\cos 2 \theta_{1}\right)\right],
\end{aligned}
$$

and

$$
\begin{aligned}
\tau_{x y}=- & (p / 2 \pi)\left[\cos 2 \theta_{1}-\cos 2 \theta_{2}\right] \\
& -(t / 2 \pi)\left[2\left(\theta_{2}-\theta_{1}\right)-\left(\sin 2 \theta_{2}-\sin 2 \theta_{1}\right)\right] .
\end{aligned}
$$

But from (1) and (2), $p$ and $t$ are given by

$$
p=q \cos \phi,
$$

and

$$
t=q \sin \phi \text {. }
$$

Substituting the above values of $p$ and $t$ in (27) and utilizing (26), we can obtain the approximate shape of the plastic zone for any given value of $\phi$. Figure 10 shows the results of such a computation for $\phi=45^{\circ}$.

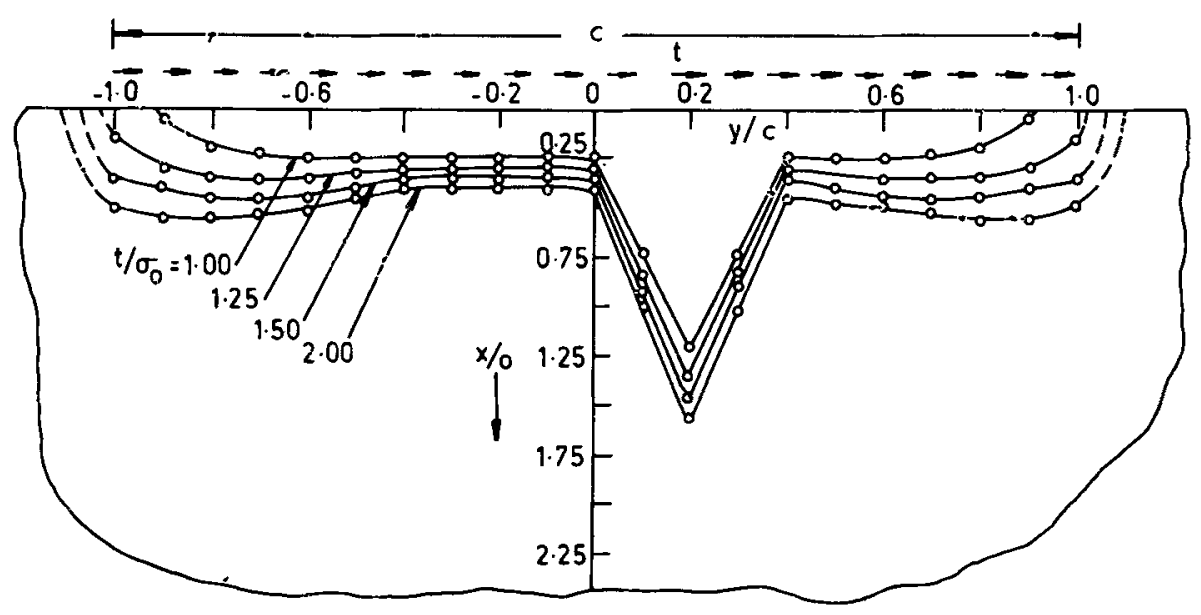

Figure 9. Variation of the plastic zone with tangential stress $t / \sigma_{0}$. 


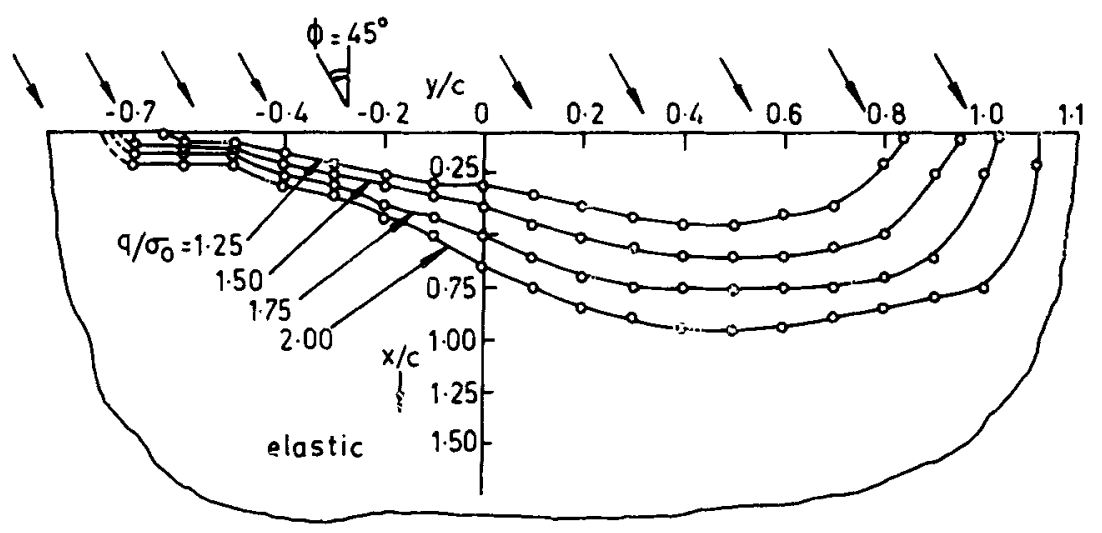

Figure 10. Variation of the plastic zone with the inclined stress $q / \sigma_{0}\left(\phi=45^{\circ}\right)$.

In this case also, it is found that the size of the plastic zone increases with increase of the applied stress $q / \sigma_{0}$. However, the shape of the plastic zone depends upon the inclination angle $\phi$. It could be closer to that of figure 5 if $\phi$ is small. As $\phi$ gradually increases, the shape gets modified due to the tangential component $t$ (2).

\section{Discussion*}

Normal concentrated edge loads on the blade of a spade produce a purely compressive stress, given by (6), in the radial direction, from the point of application of the external load. This internal stress is very large at or very near the point of application of the load but decreases in inverse proportion to the distance from the point of load application. A symmetrical region of the plate (figure 3 ) is subject to plastic deformation. As the external load increases in magnitude, so does the plastic zone.

On the other hand, a tangential concentrated line load acting on the edge of the blade of a spacie causes both compressive and tensile radial stress - compressive in the portion of the blade situated in the front of the external load and tensile behind it. As in the earlier case of the normal load, the radial stress in this case also falls off in magnitude with increase in distance from the point of application of the load. There are, in this case, two equal regions curved in shape, one in front and the other behind the point of application of the load that are subject to plastic deformation. The sizes of the plastic zones depend upon the magnitude of the external load.

Strictly speaking, concentrated loads rarely occur in actual practice. Distributed loads are far more common. This is because a concentrated load produces very large local stresses [see (6) and (21)], causing local yielding and thereby enlarging the area of contact. Uniformly distributed normal stress produces a twodimensional state of stress in the material in comparison to the uniaxial stress state produced by a single concentrated line load. The magnitude of the induced peak

\footnotetext{
* A note on the validity of the half-plane model is given in the appendix.
} 
stress is dramatically reduced, being limited to the external stress. Also, the shape of the region liable to plastic deformation is altered. The distance of the elasto-plastic boundary measured from the edge is largest at the middle of the loaded portion of the edge and diminishes more or less gradually on either side (figure 5).

Uniformly distributed tangential stress also produces a two-dimensional state of stress in the material. However, unlike in the previous case, the stress state on symmetrically located points is not identical. The maximum depth of the elasto-plastic boundary measured from the edge occurs at a section located at a distance of about $0.2 C$ ahead of the middle of the loaded portion. The magnitude of this maximum depth is greater than that produced by a normal stress of equal magnitude.

When the external stress is uniformly distributed but inclined, the effects of the normal and tangential stress superpose. At some points they tend to cancel each other, while at other points they could reinforce each other. As a result, both the size and shape of the plastic zone are modified. Computational results for the particular case, $\phi=45^{\circ}$, indicate that both the size and maximum depth of plastic zone are smaller (figures 5, 9 and 10).

While applying the above results to a"spade, one must of course remember that its blade is subject to repeated impacts of randomly varying intensity and inclination. Each successive impact load produces a corresponding stress field in the blade. If the load is extremely large, catastrophic failure or fracture of the cutting edge could take place. When the load is sufficiently large it could plastically deform a finite region of the blade. Plastic deformation, however, results in work hardening of the material and therefore a rise in the yield stress of the material. Some residual stresses are also left behind in the blade. It implies that an identical load another time will not be able to cause as much plastic deformation of the material as the earlier one. Repeated loading, however, could ultimately result in fatigue-failure of the blade.

\section{Conclusions}

1. A half-plane model for the analysis of stress in the blade of a spade has been presented. The model is valid for situations in which a concentrated line load or a uniformly distributed stress acts over a small portion of the cutting edge of the blade around its middle portion.

2. Concentrated normal and tangential external loads cause very high local stress sufficient to produce permanent strain in the material of the blade. The stress state is uniaxial in the radial direction.

3. Distributed normal and tangential external loads cause a two-dimensional stress whose peak values are restricted to much lower levels than those in the case of concentrated loads. If the external load is sufficiently large, a region of the blade of the spade is liable to plastic deformation. The size of this vulnerable region grows with increase in the magnitude of the external load and a decrease in the yield stress of the material.

4. The shape and size of the plastic zone depends upon the inclination of the external load. 
5. Successively applied identical loads do not produce identical plastic zones in the material owing to work hardening. Repeated loads, however, could lead to eventual fatigue failure.

\section{Appendix}

Validity check of the proposed model

In the present analysis, a real spade having finite dimensions has been approximated by a half-plane for stress analysis. It is pertinent to ask whether such an idealization is at all justified. For answering this question realistically, the boundary element method was used to compute the stress distribution in the spade shown in figure 11 . The boundary of the spade was divided into a fairly large number of elements upto 124. A uniform external stress was applied to one or more elements situated at the middle of the leading edge. The predictions of the present model regarding the stress distribution were compared with those of the boundary element model. It was found that the maximum difference between the two sets of results was limited to about 2 to $3 \%$ - when the length of the stressed portion of the leading edge was $1.68 \mathrm{~cm}, 2.25 \%$, when the length of the stressed portion was $0.88 \mathrm{~cm}, 2.70 \%$, and when the length of the stressed portion was $0.59 \mathrm{~cm}, 2.88 \%$. Therefore, the proposed model appears to be a very good approximation.

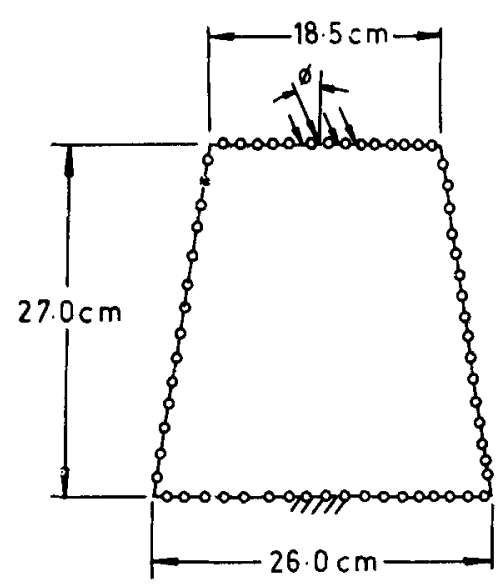

Figure 11. Figure showing boundary element model of the spade

\section{References}

Timoshenko S, Goodier J N 1951 Theory of elasticity (New York: McGraw Hill)

Saada A S 1974 Elasticity theory and applications (New York: Pergamon)

Caddle R M 1980 Deformation and fracture of solids (New Jersey: Prentice-Hall) 